\title{
Incorporating heifer feed efficiency in the Australian selection index using genomic selection
}

\author{
O. Gonzalez-Recio, ${ }^{*} \dagger^{1}$ J. E. Pryce,${ }^{*} \dagger \ddagger$ M. Haile-Mariam,${ }^{*} \dagger$ and B. J. Hayes ${ }^{*} \dagger \ddagger$ \\ *Biosciences Research Division, Department of Environment and Primary Industries, Agribio, 5 Ring Road, Bundoora, VIC 3083, Australia \\ †Dairy Futures Cooperative Research Centre, Bundoora, VIC 3083, Australia \\ łLa Trobe University, Bundoora, VIC 3083, Australia
}

\section{ABSTRACT}

The economic benefit of expanding the Australian Profit Ranking (APR) index to include residual feed intake (RFI) was evaluated using a multitrait selection index. This required the estimation of genetic parameters for RFI and genetic correlations using single nucleotide polymorphism data (genomic) correlations with other traits. Heritabilities of RFI, dry matter intake (DMI), and all the traits in the APR (milk, fat, and protein yields; somatic cell count; fertility; survival; milking speed; and temperament), and genomic correlations between these traits were estimated using a Bayesian framework, using data from 843 growing Holstein heifers with phenotypes for DMI and RFI, and bulls with records for the other traits. Heritability estimates of DMI and RFI were 0.44 and 0.33, respectively, and the genomic correlation between them was 0.03 and nonsignificant. The genomic correlations between the feed-efficiency traits and milk yield traits were also close to zero, ranging between -0.11 and 0.10 . Positive genomic correlations were found for DMI with stature (0.16) and with overall type (0.14), suggesting that taller cows eat more as heifers. One issue was that the genomic correlation estimates for RFI with calving interval (ClvI) and with body condition score were both unfavorable ( -0.13 and 0.71 respectively), suggesting an antagonism between feed efficiency and fertility. However, because of the relatively small numbers of animals in this study, a large $95 \%$ probability interval existed for the genomic correlation between RFI and ClvI $(-0.66,0.36)$. Given these parameters, and a genetic correlation between heifer and lactating cow RFI of 0.67 , inclusion of RFI in the APR index would reduce RFI by $1.76 \mathrm{~kg} /$ cow per year. Including RFI in the APR would result in the national Australian Holstein herd consuming $1.73 \times 10^{6} \mathrm{~kg}$ less feed, which is worth 0.55 million Australian dollars $(\mathrm{A} \$)$ per year and is

Received September 23, 2013

Accepted February 11, 2014.

${ }^{1}$ Corresponding author: oscar.gonzalez-recio@depi.vic.gov.au
$3 \%$ greater than is currently possible to achieve. Other traits contributing to profitability, such as milk production and fertility, will also improve through selection on this index; for example, ClvI would be reduced by $0.53 \mathrm{~d} /$ cow per year, which is $96 \%$ of the gain for this trait that is achieved without RFI in the APR.

Key words: feed efficiency, residual feed intake, genomic selection, profitability, selection index

\section{INTRODUCTION}

In the future, predicted human population growth (United Nations, Department of Economics and Social Affairs, Population Division, 2010) means that dairy industries will face the challenge of competing for grain and feed resources with other livestock industries, human consumption, and biofuel production. At the same time, global wealth is increasing, fueling a rapid global increase in demand for high-value protein, including dairy products. Improved cow feed efficiency will be important for dairy industries to both remain competitive and to meet projected demands with constrained resources. Furthermore, feed efficiency of dairy cows has a considerable effect on the profitability of dairy farms, as feed represents around $50 \%$ of total farm costs (Simm et al., 1994; Ho et al., 2005). Recognition of the importance of feed efficiency in the dairy industry has resulted in large-scale, global efforts to improve this trait (de Haas et al., 2012; Pryce et al., 2012; Hayes et al., 2013; Berry et al., 2014; Pryce et al., 2014b).

Historically, because of the expense and difficulty of measuring individual cow intakes, selection for feed efficiency has been through selection on correlated traits such as production and BW. However, it has not been possible to fully exploit variation in feed efficiency, as this requires individual measurement of intake (Pryce et al., 2014b). Genomic selection (Meuwissen et al., 2001) offers the possibility to capture additive genetic variation in feed efficiency, in addition to what is predicted by production and BW. If the accuracy of genomic breeding values for feed efficiency were high enough, animals could be selected for this trait with 
breeding based on DNA information alone. Dry matter intake and residual feed intake (RFI) are the most common traits for measuring feed efficiency in cattle (DMI is not an efficiency trait but is a component of such traits). Residual feed intake is defined as observed minus expected DMI, after adjusting for productivity. Variation in RFI is likely to be the joint effect of differences arising from basal metabolism, activity, and possible variation in deposition of specific tissues or total mass of tissue. Currently, although the contribution of each parameter contributing to RFI would be of interest, it is likely to be complicated to discern accurately, requiring large and expensive metabolic experiments.

Using high-density genotypes (630,000 genetic markers per animal) genomic EBV (GEBV) for RFI were developed by Pryce et al. (2012) using research station measurements of DMI and BW gains from both 843 growing Australian Holstein heifers and 1,034 growing New Zealand heifers, respectively. After that study, DMI phenotypes from the Australian heifers were added to phenotypes from lactating cows from the Netherlands and the United Kingdom to see if an international reference population improved genomic prediction when compared with estimates from within the country (de Haas et al., 2012). These populations gave accuracies (estimated using cross-validation) ranging between 0.42 and 0.46 for GEBV of RFI and DMI, respectively, and genomic heritability estimates ranging from 0.34 to 0.58. Recently, Pryce et al. (2014a) validated the equations developed by de Haas et al. (2012) and Pryce et al. (2012) in an independent population of lactating cows, and reported accuracies of GEBV for DMI of up to 0.72 , albeit with a large standard error. Importantly, Pryce et al. (2014a) estimated the genetic correlation between RFI in growing heifers and RFI in lactating cows to be 0.67 .

These accuracies of GEBV for RFI and DMI are promising, suggesting that genetic gains could be made through genomic selection. However, optimizing selection for profitability requires that feed efficiency is included as part of a selection index that includes all other traits contributing to profit. To evaluate the responses to selection using selection index theory requires genetic correlation estimates of RFI or DMI with other traits. In fact, RFI could be more straightforward to include in a selection index than DMI, as it is expected to have correlations that are not different from zero with several other traits, as RFI is by definition already adjusted for growth, weight, and production.

The aims of this study were to (1) estimate genomic correlations between 2 traits that contribute to feed efficiency traits for heifers, DMI, and RFI, with other traits of economic importance, including milk yield, conformation, fertility and workability traits, and (2) use these correlations to investigate the consequences of selecting on an expanded version of the Australian Profit Ranking (APR) index that includes RFI as a measure of feed efficiency.

\section{MATERIALS AND METHODS}

This section first describes the phenotypes and genotypes used in the study and the relative weightings for phenotypes from males and females. Then, the models to estimate genomic correlations between feed conversion traits and other traits in the index are described in a Bayesian framework. Finally, selection index theory is used to predict responses to selection for traits in the APR index that incorporates RFI, in addition to an overall economic response to selection.

\section{Phenotypes for Feed Conversion Efficiency}

In this study, 843 calves were available with DMI recorded daily during 3 experiments carried out over 2 yr (2009 and 2010) in Rutherglen, Victoria, Australia. Calves were offered alfalfa (Medicago sativa) cubes ad libitum, which is somewhat similar to the predominant dairy feed available in Australia (grass pasture), but has the advantage of consistent nutritive values. Body weight was measured at the beginning of the trial and weekly thereafter. A comprehensive description of the experiments is provided in Williams et al. (2011). On average, 63 daily records for DMI were available per cow.

Residual feed intake was calculated using a mixed linear model on daily measurements of DMI as follows:

$$
\begin{aligned}
& y_{i j k l m n}=\mu+\operatorname{farm}_{i}+\operatorname{trial}_{j}+\beta_{1} \operatorname{age}_{k}+\beta_{2} \operatorname{age}_{k}^{2} \\
& +\beta_{3} \mathrm{BW}_{l}+\beta_{4} \Delta \mathrm{BW}_{m}+\text { cow }_{n}+e_{i j k l m n},
\end{aligned}
$$

where $y_{i j k l m n}$ is the daily DMI observation for a given animal $n ; \mu$ is the overall mean; farm $_{i}$ is a categorical fixed effect of the farm where the calf was born $(i=$ $1-35) ; \operatorname{trial}_{j}(j=1-3)$ is a categorical effect of the feed efficiency trial (as heifers were taken through the trials in 3 cohorts); age $_{k}$ is a continuous fixed effect of the age of the calf at the day of the measurement, with regression coefficient $\beta_{1}$; $\operatorname{age}_{k}^{2}$ is a continuous effect of the quadratic age of the calf, with regression coefficient $\beta_{2}$; $\mathrm{BW}_{l}$ is a continuous fixed effect of the $\mathrm{BW}(\mathrm{kg})$, with regression coefficient $\beta_{3} ; \Delta \mathrm{BW} \mathrm{W}_{m}$ is a continuous fixed effect of the weekly variation of BW $(\mathrm{kg})$, with regression coefficient $\beta_{4} ; \operatorname{cow}_{n}$ is the random animal effect; and $e_{i j m l m n}$ is the random residual effect. After fitting the model, the estimate of the random animal effect was considered as the RFI phenotype for that cow in 
Table 1. Number of animals with phenotypes per trait, with respective phenotypic means and SD

\begin{tabular}{lcccc}
\hline Trait $^{1}$ & $\begin{array}{c}\text { Cows with } \\
\text { phenotype }\end{array}$ & $\begin{array}{c}\text { Sires with } \\
\text { phenotype }\end{array}$ & Mean & SD \\
\hline DMI (kg) & 843 & 0 & 100 & 13.4 \\
RFI (kg) & 843 & 0 & 100 & 14.4 \\
MY (kg) & 304 & 87 & 1,534 & 624 \\
FY (kg) & 304 & 87 & 38 & 22 \\
PY (kg) & 304 & 87 & 46 & 18 \\
ClvI (d) & 304 & 87 & 108 & 27 \\
SCC (cells/mL) & 299 & 87 & 100 & 24 \\
STAT (score) & 304 & 87 & 104 & 10 \\
OType (score) & 304 & 87 & 107 & 12 \\
BCS (score) & 304 & 87 & 100 & 4 \\
SURV (d) & 304 & 87 & 114 & 21 \\
MSpeed (score) & 298 & 87 & 92 & 26 \\
TEMP (score) & 298 & 92 & 27 \\
\hline RFI & & & 97
\end{tabular}

${ }^{1} \mathrm{RFI}=$ residual feed intake; $\mathrm{MY}=$ milk yield; $\mathrm{FY}=$ fat yield; $\mathrm{PY}=$ protein yield; $\mathrm{ClvI}=$ calving interval; $\mathrm{STAT}=$ stature; OType $=$ overall type; SURV $=$ survivability; MSpeed $=$ milking speed; TEMP $=$ temperament.

the genomic analyses. The phenotype of calf DMI was determined as the average DMI over the measurement period. Both DMI and RFI were scaled to have a mean of 100 and a standard deviation of 10 , as this improves convergence when parameters (heritability and covariance) were estimated in the Bayesian framework as described below.

The phenotypes for traits other than DMI and RFI [milk yield, fat yield, protein yield, calving interval (ClvI), SCC, stature, overall type, BCS, survival, milking speed, and temperament] were daughter trait deviations for 87 sires that were within 3 generations of the pedigree of each of the heifers in the analysis and trait deviations for the heifers themselves, when they became lactating cows. Weights to account for different information content were derived following Garrick et al. (2009) to combine information from 87 sires and 843 cow trait deviations. Cow trait deviations were weighted $\left(w_{T D}\right)$ as follows:

$$
w_{T D}=\frac{1-h^{2}}{c h^{2}+\frac{1+t(n-1)}{n}-h^{2}},
$$

whereas sire daughter trait deviations were weighted $\left(w_{D T D}\right)$ as follows:

$$
w_{D T D}=\frac{1-h^{2}}{c h^{2}+\frac{4-h^{2}}{p}},
$$

where $h^{2}$ and $t$ are the heritability and repeatability of the corresponding trait, respectively, $c$ is the proportion of genetic variance not explained by markers (set fixed at 0.20 ) as estimated by Haile-Mariam et al. (2013b), $n$ is the number of repeated records for the cow, and $p$ is the number of progeny daughters of the sire. Weights for DMI and RFI for heifers were set to 1 (e.g., 1 record). Table 1 shows the number of animals with phenotypes per trait, with respective phenotypic means and standard deviations.

\section{Genotypes}

The 843 heifers were genotyped using the Bovine HD BeadChip (Illumina Inc., San Diego, CA). The same quality control processes as previously described by Erbe et al. (2012) and Pryce et al. (2012) were applied to the genotype data. The edits included overall call rates of greater than $95 \%$ and the use of the Illumina GenCall score, which describes the performance of genotyping each SNP in each individual. Genotype calls with GenTrain score (GenCall) $>0.6$ are high quality (Erbe et al., 2012). Single nucleotide polymorphisms were also excluded if they were unmapped or mismapped, had duplicate positions, or were on the $\mathrm{Y}$ chromosome.

In total, 632,002 SNP were kept for the analyses. In addition, the genotypes of 87 sires related to the cows (within 3 generations) were also included. Sires were genotyped with the Bovine 50K BeadChip (Illumina Inc.), and were imputed to high density $(632,002)$ using the 843 heifers and 940 other animals (mainly Australian Holstein bulls) as the reference population using BEAGLE 3 software (Browning and Browning, 2009). In total, 960 genotyped animals were used to construct the genomic relationship matrix among the animals, using all markers kept after editing, as described by Yang et al. (2010). 


\section{Estimates of Genomic Parameters}

Estimates of the heritabilities and trivariate genomic relationship for feed efficiency and residual feed intake with other traits were implemented using multitrait models in a Bayesian framework as follows.

Phenotypic data on all individuals is represented by matrix $\mathbf{Y}$, containing the vectors with the corresponding phenotypes for DMI, RFI, and the trait $k$ being analyzed, as $\mathbf{Y}=\left(\mathbf{y}_{d m i}, \mathbf{y}_{r f i}, \mathbf{y}_{k}\right)$. The phenotypes of individual $i$ are in the vector $\mathbf{y}_{i}=\left(\eta_{i, d m i}, \eta_{i, r f i}, \eta_{i, k}\right)$, with $\eta_{i}$, being its observed or augmented record for DMI, RFI, or trait $k$. The augmented record is the estimated phenotype given the corresponding effect estimates, if the trait record was missing. A vector $\boldsymbol{\delta}_{\mathrm{i}}=\left(\delta_{i, d m i}\right.$, $\delta_{i, r f i}, \delta_{i, k}$ ) denoted missing (0) or observed (1) record for animal $i$.

The following linear model was assumed:

$$
\mathbf{y}=\mathbf{1}^{\prime} \mu+\mathbf{Z u}+\mathbf{e}
$$

where $\mathbf{y}$ are the phenotypes as described before, $\mathbf{Z}$ is an incidence matrix relating observations to individuals in the genomic relationship matrix, and $\mathbf{u}$ are the genomic breeding values assumed to be distributed as $\mathbf{u} \mid \mathbf{G} \sim N\left(0, \mathbf{G} \otimes \mathbf{V}_{q}\right)$, where $\mathbf{G}$ is the genomic relationship matrix and

$$
\mathbf{V}_{g}=\left[\begin{array}{ccc}
\sigma_{d m i}^{2} & \sigma_{d m i, r f i} & \sigma_{d m i, k} \\
& \sigma_{r f i}^{2} & \sigma_{r f i, k} \\
S & & \sigma_{k}^{2}
\end{array}\right]
$$

is the genomic (co)variance matrix between DMI, RFI, and the trait $k$, where $S$ indicates symmetric. The $\mathbf{V}_{g}$ are defined as genomic (co)variances ( $\sigma$ terms) because they are obtained from the assumption that covariances between individuals are driven by the genomic relationship matrix. Then, e are the residuals assumed to be distributed as $\mathbf{e} \mid \mathbf{R} \sim N\left(0, \mathbf{R}=\mathbf{W} \otimes \mathbf{V}_{e}\right)$, where $\mathbf{R}$ is the residual covariance structure matrix, with $\mathbf{W}$ being a diagonal matrix containing the corresponding weights in the diagonal, and

$$
\mathbf{V}_{e}=\left[\begin{array}{ccc}
\sigma_{e(d m i)}^{2} & \sigma_{e(d m i, r f i)} & \sigma_{e(d m i, k)} \\
& \sigma_{e(r f i)}^{2} & \sigma_{e(r f i, k)} \\
S & & \sigma_{e(k)}^{2}
\end{array}\right]
$$

is the residual (co)variance matrix. Inverted Wishart distributions were assigned as informative priors to $\mathbf{V}_{g}$ and $\mathbf{V}_{e}$ to assist in the convergence.
Assuming that conditionally on model parameters $\boldsymbol{\theta}$ $=\left(\mu, \mathbf{u}, \mathbf{V}_{g}, \mathbf{V}_{e}\right)$, missing values are noninformative and independent, the augmented posterior distribution is given by

$$
p(\boldsymbol{\theta}, \boldsymbol{\eta} \mid \mathbf{y}, \boldsymbol{\delta}) \propto p(\mathbf{y}, \boldsymbol{\delta}, \boldsymbol{\eta} \mid \boldsymbol{\theta}) p\left(\mathbf{u} \mid \mathbf{V}_{g}\right) p\left(\mathbf{e} \mid \mathbf{V}_{e}\right) p\left(\mathbf{V}_{g}\right) p\left(\mathbf{V}_{\mathrm{e}}\right),
$$

where

$$
\begin{aligned}
& p(\mathbf{y}, \boldsymbol{\delta}, \boldsymbol{\eta} \mid \boldsymbol{\theta}) \propto \\
& \prod_{i=1}^{N}|\mathbf{R}|^{-1 / 2} \exp \left(-\frac{1}{2}\left\{\left[\mathbf{y}_{i}-E\left(\mathbf{y}_{i}\right)\right]^{\prime \delta_{i}} \mathbf{R}^{-1}\left[\mathbf{y}_{i}-E\left(\mathbf{y}_{i}\right)\right]^{\delta_{i}}\right\}\right),
\end{aligned}
$$

where $N=$ the number of records and $E=$ expectation. The sampling model for missing observations was standardized as

$$
\boldsymbol{\eta} \mid \boldsymbol{\theta}, \boldsymbol{\delta}=0 \sim N\left(\mathbf{1}^{\prime} \mu+\mathbf{Z u}, \mathbf{R}\right) .
$$

Posterior distributions of the parameters of interest were estimated using a Gibbs sampling algorithm. Visual inspections of the running means and trace plots were used to assess burn-in period and convergence.

The analyses were based on 50,000 iterations after discarding the first 10,000 as burn-in. The effective sample size and the Monte Carlo standard error of the heritability and genomic correlations estimates were calculated as described by Geyer (1992).

\section{Selection Index}

A multitrait selection index was used to quantify selection responses for the APR index adapted to include RFI. The current APR index includes the following traits: milk, fat and protein yield, mastitis resistance (predicted using SCC), fertility (ClvI), liveweight, survival, temperament, and milking speed (http://www. adhis.com.au/v2/downv2.nsf/0/15cea0359dbe86e4ca25 $774 \mathrm{c} 0015 \mathrm{f} 192$ ?open) and has previously been described by Pryce et al. (2009) and Bell et al. (2013).

Selection index theory, implemented using software developed by J. van der Werf (http://www-personal. une.edu.au/ jvanderw/software.htm), was used to predict annual responses to selection using the current APR as well as on the proposed alternative indices, given phenotypic information on their progeny, estimates of genomic parameters, and economic values of traits in the aggregate genotype (Hazel, 1943). As far as possible, the selection index model was designed to reflect current practices by the Australian Dairy Herd Improvement Scheme (ADHIS). For example, no phenotypic measurement was assumed to be available for 
liveweight and RFI, and indirect selection of liveweight and survival were based on predictive information from type traits. Residual feed intake was assumed to be available only as a GEBV for heifers with an accuracy of prediction of 0.40 for heifer RFI and 0.25 for cow RFI (Pryce et al., 2012, 2014a). The genetic correlation between heifer and lactating cow RFI was taken as 0.67 , as estimated by Pryce et al. (2014a). For all other traits, breeding values were assumed to be estimated using single-trait models, (i.e., in accordance with ADHIS practices). The number of daughters per sire was assumed to be 75 for milk production traits, fertility, and survival; 70 for SCC; and 60 for milking speed and temperament. The model included a total of 35 traits (i.e., the traits in APR and type traits evaluated by ADHIS) and covariances between these traits were taken into account when deriving optimal index weights by using a (co)variance matrix of traits in the index.

The current version of APR published by ADHIS (http://www.adhis.com.au/v2/downv2.nsf/0/15cea0 359dbe86e4ca25774c0015f192?open) includes an artificially increased weighting on fertility and survival, as previously described by Pryce et al. (2009). This increase ensures that the annual rate of genetic gain in fertility and survival would be more than would occur if their derived economic index weights were used. A 2-step approach was, therefore, used to derive index weights: (1) the index weights (b) were calculated using selection index theory [i.e., as $\mathbf{b}=\mathbf{P}^{-1} \mathbf{G a}$, where $\mathbf{P}$ is the phenotypic (co)variance matrix, $\mathbf{G}$ is the genetic/ genomic (co)variance matrix, and $\mathbf{a}$ is the vector of economic values] and (2) the index weights for survival and fertility were multiplied by 1.5 and 3 , respectively, and then selection responses were calculated using these index weights. The calculation of economic values for traits in the current APR and for RFI are described by Bell et al. (2013). The economic value for RFI, estimated by Bell et al. (2013) was, in Australian dollars $(\mathbf{A} \$),-\mathrm{A} \$ 102.61 /$ daily $\mathrm{kg}$ of RFI per cow per year, meaning that decreasing RFI by $1 \mathrm{~kg} / \mathrm{d}$ would cause profit to increase by $\mathrm{A} \$ 102.61$ per cow per year, assuming all other values were held constant.

Response to selection of the index was assumed to be 0.25 genetic standard units averaged across the 4 selection pathways, as could be achieved in a well-organized progeny-testing scheme (Pryce et al. (2010). Then, annual selection responses and the economic impact of the inclusion of RFI in the extended APR were compared with the current APR. A sensitivity analysis of the index was also conducted to take into account the uncertainty of the genomic correlation estimates between fertility and RFI. Although the standard deviations of the mean correlation estimates were reasonably large for all traits, the sensitivity of response to selection for fertility is particularly important because of the close association between RFI and energy balance (Pryce et al., 2014b), and the high relative emphasis on fertility in the APR. Therefore, 4 scenarios were devised to understand the consequences of selection including RFI:

(1) Current APR without RFI (i.e., the economic value for RFI was 0 ); however, genomic correlations between RFI and APR traits were included, so that the correlated response of RFI to selection on APR could be examined;

(2) Extended APR that included RFI weighted by its economic value and the respective genomic correlations with other traits.

Further analyses were conducted to assess sensitivity of selection response to the genomic correlation between RFI and ClvI:

(3) Similar to (2) but with the genomic correlation between RFI and ClvI set to 0;

(4) Similar to (2) but with the genomic correlation between RFI and CI set to -0.5 .

\section{RESULTS AND DISCUSSION}

\section{Genomic Parameters}

Heritability estimates of DMI and RFI estimated using genomic relationships were 0.44 and 0.33 , respectively (Table 2). In accordance with previously published studies (e.g., Arthur et al., 2001; de Haas et al., 2012), genetic variation was considerable for these traits, suggesting that selection to improve these traits is possible. For other traits, heritability estimates were as expected, except for stature (0.70), which was higher than reported in other studies, where typically the heritability estimates are around 0.38 (Haile-Mariam et al., 2014), probably because these animals were raised in very particular conditions in experimental farms and also because of the reduced sample size.

The posterior mean estimate of the genomic correlation between DMI and RFI was not significantly different from zero (0.03, with an associated standard error of 0.07 ). The posterior means of the genomic correlation estimates between DMI or RFI and each of the traits in the index are presented in Table 3 . The genomic correlations between DMI, RFI, and milk production traits were not different from zero, ranging between -0.10 and 0.11 , with mean estimates closer to zero for RFI, suggesting that these feed efficiency traits in heifers are not correlated with cow milk yield. A positive genomic correlation was found between stature and DMI (0.16), 
Table 2. Means of the posterior distribution of the heritability estimate $\left(\mathrm{h}^{2}\right)$ for each of the traits in the index, with the respective SD, Monte Carlo SE (MCse), and effective sample size (ESS)

\begin{tabular}{lcccc}
\hline Trait $^{1}$ & $\mathrm{~h}^{2}$ & SD & MCse & ESS \\
\hline DMI & 0.44 & 0.07 & 0.01 & 232.1 \\
RFI & 0.33 & 0.05 & 0.01 & 234.8 \\
MY & 0.43 & 0.07 & 0.01 & 267.1 \\
FY & 0.41 & 0.06 & 0.01 & 259.6 \\
PY & 0.44 & 0.05 & 0.01 & 248.3 \\
ClvI & 0.03 & 0.02 & 0.01 & 174.6 \\
SCC & 0.18 & 0.11 & 0.01 & 228.3 \\
STAT & 0.70 & 0.03 & 0.01 & 206.4 \\
OType & 0.33 & 0.12 & 0.01 & 211.6 \\
BCS & 0.10 & 0.07 & 0.01 & 191.0 \\
SURV & 0.16 & 0.06 & 0.01 & 135.4 \\
MSpeed & 0.24 & 0.07 & 0.01 & 152.2 \\
TEMP & 0.26 & 0.07 & 0.01 & 170.7
\end{tabular}

${ }^{1} \mathrm{RFI}=$ residual feed intake; $\mathrm{MY}=$ milk yield $; \mathrm{FY}=$ fat yield; $\mathrm{PY}$ $=$ protein yield $;$ ClvI = calving interval; STAT = stature; OType = overall type; SURV = survivability; MSpeed = milking speed; TEMP $=$ temperament.

indicating that taller cows ate more as heifers. A strong negative genomic correlation was also found between stature and RFI (-0.5), interpreted as taller animals having greater feed efficiency as heifers. Genomic correlations between overall type and feed efficiency traits were positive, contrary to what was expected, because overall type is correlated to stature (Haile-Mariam et al., 2014), and this may imply larger maintenance requirements. However, most of the correlation estimates presented have large posterior standard deviations; they should, therefore, be interpreted with care.

An important aspect to take into account when selecting for feed efficiency is potential antagonism with fertility (Shaffer et al., 2011; Vallimont et al., 2013; Pryce et al., 2014b). The genomic correlations between ClvI and DMI and RFI were 0.26 and -0.13 , respectively. The estimate of the genomic correlation between

Table 3. Means (SD in parentheses) of the posterior distribution of the genomic correlation estimate between DMI or residual feed intake (RFI) and each of the traits in the index

\begin{tabular}{lrr}
\hline Trait $^{1}$ & \multicolumn{1}{c}{ DMI } & \multicolumn{1}{c}{ RFI } \\
\hline MY & $0.10(0.11)$ & $0.07(0.08)$ \\
FY & $-0.03(0.10)$ & $0.02(0.07)$ \\
PY & $-0.11(0.08)$ & $0.03(0.07)$ \\
ClvI & $0.26(0.15)$ & $-0.13(0.25)$ \\
SCC & $-0.24(0.29)$ & $0.17(0.23)$ \\
STAT & $0.16(0.14)$ & $-0.50(0.22)$ \\
OType & $0.14(0.19)$ & $0.23(0.31)$ \\
BCS & $0.37(0.32)$ & $0.71(0.32)$ \\
SURV & $-0.22(0.28)$ & $0.19(0.26)$ \\
MSpeed & $-0.14(0.19)$ & $-0.27(0.27)$ \\
TEMP & $-0.21(0.17)$ & $0.01(0.21)$ \\
\hline
\end{tabular}

${ }^{1} \mathrm{MY}=$ milk yield; $\mathrm{FY}=$ fat yield $; \mathrm{PY}=$ protein yield; $\mathrm{ClvI}=$ calving interval; STAT $=$ stature; OType $=$ overall type; SURV $=$ survivability; MSpeed $=$ milking speed; TEMP $=$ temperament.
RFI and ClvI was unfavorable (i.e., more efficient heifers are less fertile as cows). Heifers eating more tend to gain condition score, presumably by converting more feed into larger amounts of fat reserves (the genomic correlation estimate between BCS and DMI was 0.37) and this is likely to be highly inefficient because the mobilization of fat tissue requires larger amounts of energy (the correlation between BCS and RFI was 0.7: the larger the BCS, the less efficient). However, the unfavorable correlation between RFI and ClvI was smaller than the unfavorable correlation between ClvI and milk yield (0.41; Haile-Mariam et al., 2013a).

The posterior distribution of the heritability estimates showed standard deviations of 0.07 and 0.05 for DMI and RFI, with small Monte Carlo standard errors (0.01) for the posterior means (Table 2). For other traits, the standard deviations ranged between 0.02 for ClvI and 0.12 for overall type, with Monte Carlo standard errors of 0.01 . Larger standard deviations were found for the posterior estimates of genomic correlations, as expected, ranging between 0.07 between RFI and protein yield and 0.32 between BCS and DMI or RFI. This is likely to be because of the small number of sires with daughter trait deviation information. Although weights were included in the analyses, the uncertainty of the estimates could be considerable, as suggested by the standard deviations of the posterior estimates. For instance, the genomic correlations between feed efficiency, measured as RFI, and traits such as ClvI, SCC, overall type, survival, milking speed, or temperament all included zero in the confidence interval of the genomic correlation. However, the Monte Carlo standard error for the posterior mean of the genomic correlation estimates ranged from 0.01 to 0.06 . The effective sample sizes for the Monte Carlo Markov chain were reasonable, ranging from 124 to 249 (Table 4), which is the number of totally independent samples extracted from the Markov chain Monte Carlo algorithm.

It should be pointed out that these correlations were estimated under experimental conditions; for example, DMI and RFI phenotypes were derived from ad libitum intakes of Lucerne cubes, rather than competitive grazing at pasture. Unfortunately very little information exists in the literature comparing DMI and RFI in an experimental setting to grazing conditions (which is likely to reflect the difficulty of doing such an experiment).

\section{Selection Index}

The response to selection in units and Australian dollars for each trait in each of the 4 indices (scenarios) are presented in Table 5. The current APR was used as a comparison, and selection on this index results 
Table 4. Monte Carlo SE and effective sample size of the posterior distribution of the genomic correlation estimate between DMI or residual feed intake (RFI) and each of the traits in the index

\begin{tabular}{|c|c|c|c|c|}
\hline \multirow[b]{2}{*}{ Trait $^{1}$} & \multicolumn{2}{|c|}{ Monte Carlo SE } & \multicolumn{2}{|c|}{ Effective sample size } \\
\hline & DMI & RFI & DMI & RFI \\
\hline MY & 0.01 & 0.01 & 242.8 & 240.3 \\
\hline FY & 0.01 & 0.01 & 249.0 & 235.8 \\
\hline PY & 0.01 & 0.01 & 242.9 & 239.6 \\
\hline ClvI & 0.06 & 0.05 & 172.0 & 173.0 \\
\hline $\mathrm{SCC}$ & 0.04 & 0.03 & 226.1 & 227.8 \\
\hline STAT & 0.01 & 0.03 & 249.6 & 204.0 \\
\hline OType & 0.02 & 0.03 & 229.7 & 202.9 \\
\hline BCS & 0.02 & 0.03 & 200.5 & 193.1 \\
\hline SURV & 0.03 & 0.03 & 125.6 & 132.1 \\
\hline MSpeed & 0.02 & 0.03 & 151.8 & 134.8 \\
\hline TEMP & 0.02 & 0.02 & 156.8 & 144.5 \\
\hline
\end{tabular}

${ }^{1} \mathrm{MY}=$ milk yield $; \mathrm{FY}=$ fat yield; $\mathrm{PY}=$ protein yield; $\mathrm{ClvI}=$ calving interval; STAT $=$ stature; OType $=$ overall type; SURV $=$ survivability; MSpeed $=$ milking speed; TEMP $=$ temperament.

in an increase in profit of $\mathrm{A} \$ 22.73$ per cow per year. Adding RFI to the index resulted in a $1.76 \mathrm{~kg} / \mathrm{cow}$ per year improvement in RFI and increased the rate of genetic gain per cow per year to $\mathrm{A} \$ 23.28$. Therefore, inclusion of heifer RFI in the APR would increase profit by $2.4 \%$, with similar genetic gain in production, an increase in liveweight and, importantly, a reduction in the annual increase in RFI of $67 \%$ (compared with the current APR). In summary, the proposed APR + RFI index would result in selection of efficient heifers that have reduced feed intake with only a small impact on production. Part of the reason for the relatively small increase in profit is that APR already accounts for feed costs by having milk production in the index and penalizing larger liveweight, as heavier cows have larger maintenance costs. The economic values for each trait in the current version of APR (implemented by ADHIS) includes marginal feed costs (Bell et al., 2013). However, individual variation in actual versus predicted feed intake is currently not included in APR, which is why RFI is an appropriate trait to include in a multitrait selection index to improve overall profitability. It must be pointed out that the rates of genetic gain showed here assume complete uptake of the APR index by all farmers. In fact, actual rates of genetic gain are much lower [close to $\$ 8 / \mathrm{yr}$ in APR (D. Abernathy, ADHIS, Melbourne, VIC, Australia, personal communication)], indicating that uptake of the APR is currently suboptimal.

Some authors have suggested that RFI needs to be incorporated cautiously in breeding goals, as it is unfavorably correlated with negative energy balance and liveweight (e.g., Veerkamp et al., 2000). Our results support this, as weak but unfavorable genomic correlations existed between RFI and fertility. In fact, the most logical way of selecting for improved RFI is within a multitrait selection index, such as the APR, rather than single-trait selection, which could lead to

Table 5. Annual responses to selection [in units of measurement and in Australian dollars (A\$)] for the Australian Profit Ranking (APR) index, APR plus residual feed intake (eAPR), and eAPR_0, where the genomic correlation between residual feed intake (RFI) and fertility was assumed to be zero and eAPR_ -0.5 where the genomic correlation between RFI and fertility was -0.5

\begin{tabular}{|c|c|c|c|c|c|c|c|c|}
\hline Item & \multicolumn{2}{|c|}{ Scenario 1} & \multicolumn{2}{|c|}{ Scenario 2} & \multicolumn{2}{|c|}{ Scenario 3} & \multicolumn{2}{|c|}{ Scenario 4} \\
\hline \multicolumn{9}{|l|}{ Trait } \\
\hline Milk yield (L) & 49.01 & -2.58 & 49.20 & -2.59 & 48.28 & -2.54 & 51.90 & -2.74 \\
\hline Protein yield (kg) & 2.05 & 11.70 & 1.99 & 11.92 & 1.96 & 11.73 & 2.08 & 12.47 \\
\hline Fertility (d) & -0.55 & 1.66 & -0.53 & 1.61 & -0.57 & 1.71 & -0.43 & 1.29 \\
\hline $\operatorname{SCC}(\%)$ & -2.72 & 0.71 & -2.48 & 0.65 & -2.46 & 0.64 & -2.55 & 0.67 \\
\hline Liveweight $(\mathrm{kg})$ & 0.22 & -0.18 & 0.49 & -0.41 & 0.48 & -0.41 & 0.52 & -0.44 \\
\hline Milking speed (1-5) & -0.15 & 0.27 & -0.13 & 0.23 & -0.13 & 0.23 & -0.14 & 0.23 \\
\hline Temperament $(1-5)$ & -0.35 & 0.94 & -0.36 & 0.96 & -0.35 & 0.95 & -0.37 & 1.00 \\
\hline RFI $(\mathrm{kg} / \mathrm{yr})$ & 2.61 & -0.73 & 0.85 & -0.24 & 0.59 & -0.17 & 1.35 & -0.38 \\
\hline Total profit $(\mathrm{A} \$)$ & & 22.73 & & 23.28 & & 23.10 & & 23.74 \\
\hline
\end{tabular}


undesirable correlated responses. The most probable value of the correlation between ClvI and RFI in our population was -0.13 . When this correlation was used between ClvI and RFI, the response to selection for ClvI in the extended APR was $-0.53 \mathrm{~d} /$ cow per year (Table 4); that is, fertility continues to improve at $96 \%$ of the rate of the current APR index. The index optimizes selection responses and greater profit is achieved by reducing RFI instead of increasing solids in milk. The effect of adding RFI to the APR on survivability, milking speed, and temperament would be negligible.

Given that fertility is currently a trait of worldwide concern in dairy production, we investigated the effect of varying the correlation between ClvI and RFI on response to selection for these traits. The $95 \%$ highest probability interval of the estimate of this correlation was between -0.66 and 0.36 . Two scenarios were investigated; a favorable scenario with the genomic correlation of ClvI with RFI equal to zero, and an unfavorable scenario of -0.5 for this correlation. Index weights were recalculated for each scenario. With these assumptions, response to selection in ClvI would be -0.57 and -0.43 $\mathrm{d}$, respectively (Table 5 ). So if the correlation between RFI and fertility is actually -0.5 (instead of -0.13 ; Table 3), then the selection response in fertility would be reduced by $22 \%$ compared with the current index. However, importantly, genetic progress in fertility will still be made with a reduction in ClvI of $0.43 \mathrm{~d} /$ cow per year and a $4 \%$ increase in total profit genetic gain relative to the current APR due to the expected increase in production traits.

We also assessed the extent of reranking of bulls that would occur if the APR + RFI index were adopted. The proposed index would result in minimal reranking of animals with a Pearson and Spearman correlation of 0.99 (Figure 1). However, some reranking would be expected for 44 sires within the top 50 for the current APR (Figure 2).

We also investigated the distribution of bulls with the lowest RFI GEBV (most efficient) across APR values (without RFI added), to investigate whether a trend existed for RFI to be associated with high or low APR (Figure 3A). The bulls with lower (more efficient) EBV for RFI had APR values across all percentiles of APR, meaning that an animal that had a high GEBV value for RFI was not necessarily the most profitable one, as this depends on EBV for other traits as well. Similarly, low-RFI GEBV bulls from a feeding perspective were not necessarily those with the highest EBV for production. As shown in Figure 3B, the top 200 animals for RFI GEBV are distributed along all percentiles for fat + protein EBV. Figure 3C shows that small, intermediate, and large animals may be efficient from a feeding perspective. All these results are in contrast to the association between high protein EBV and high APR (Figure 3D; i.e., the top 200 bulls for protein are more likely to rank in the higher percentiles of APR).

The main limitation of this study was that heifer RFI was used instead of cow RFI. We used a value of 0.67 for the genetic correlation between these 2 traits, estimated by Pryce et al., (2014a). This value had a large standard error, given the small numbers of lactating cows used in that study. However, the value is similar to estimates from other experiments (e.g., 0.58 from Nieuwhof et al. 1992). The correlation between these traits is highly unlikely to be 1 , as efficiency in heifers is associated with maintenance and growth, whereas feed efficiency in mature cows is mainly related to maintenance and milk production requirements. Further, lactating cows can appear efficient by metabolism of body tissue. Heifer RFI and lactating cow RFI are likely to be more correlated during the first lactation, being a transitional stage in which cows are still growing at the same time as producing milk [however, the estimate from Pryce et al. (2014a) comes from cows mainly in second-and-later lactations]. First lactation is an important and critical period for the longevity of the cow, as any disease or severe negative energy balance may affect permanently the productive performance of the animal. Ideally, future modifications of the APR + RFI index should include both heifer and cow RFI to properly account for estimated genomic correlations between these traits. This will likely lead to further improvement in feed efficiency and profit response due

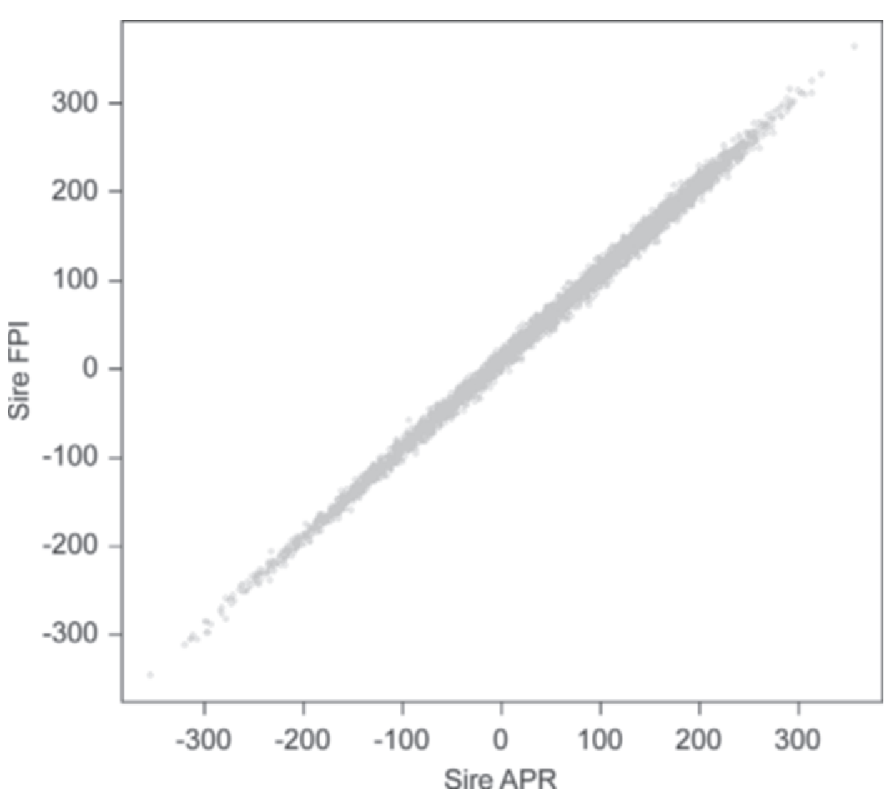

Figure 1. Relationship between the current Australian Profit Ranking (APR) index, and the extended index (FPI) including residual feed intake for all progeny-tested sires in Australia. 


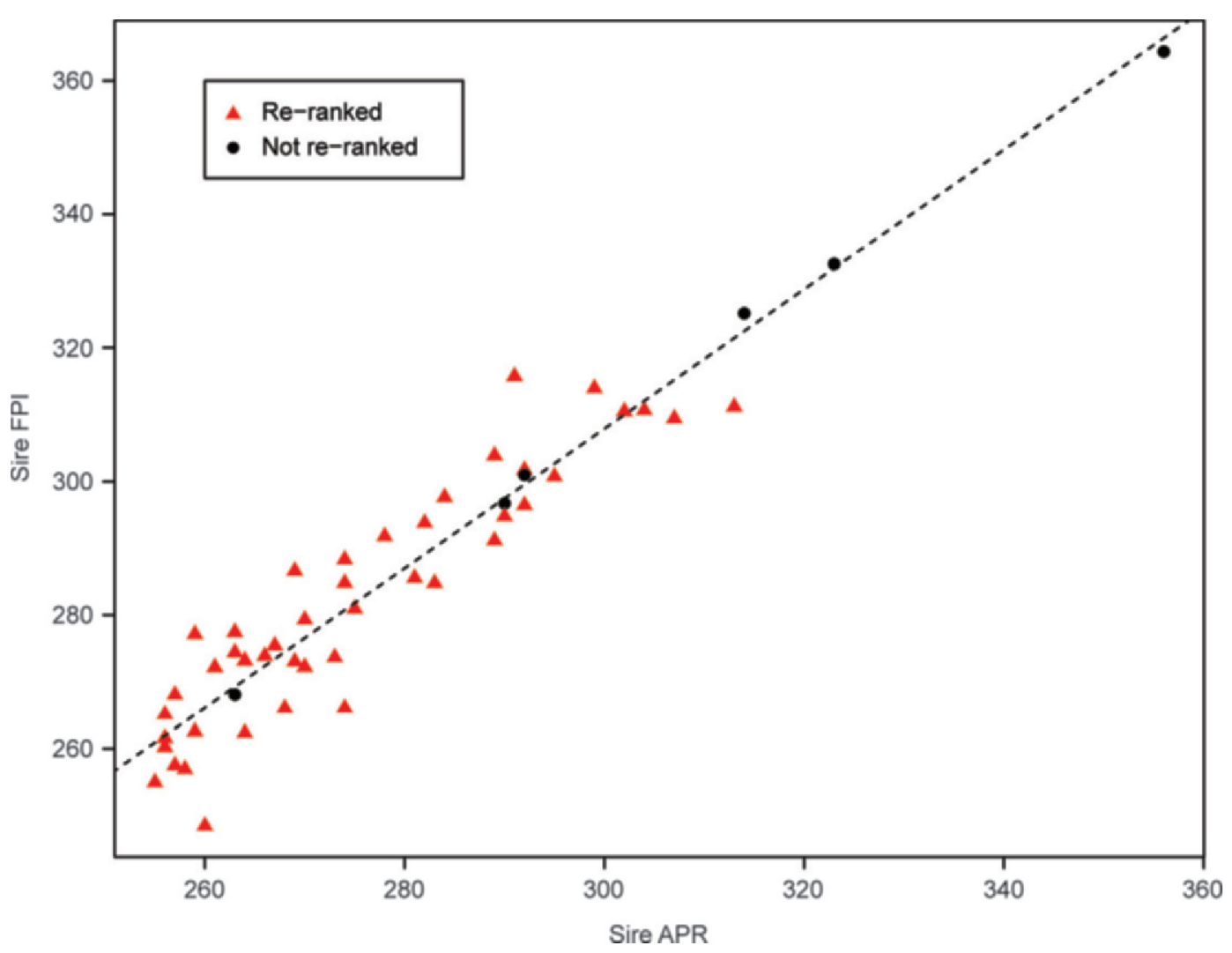

Figure 2. Expected reranking in the top 50 bulls in the current Australian Profit Ranking (APR) index, with the inclusion of residual feed intake in the index (FPI). Color version available in the online PDF.

to more accurate parameters. This requires a suitable number of records, which are currently unavailable in Australia, but may be feasible through further research and collaboration with other research groups. The phenotypic standard deviation of RFI used in the selection index calculations was 0.1 , which was from the heifer study of Pryce et al. (2014a). However, the variation in cow RFI is considerably larger: the phenotypic standard deviation of RFI is more than double heifer RFI (Macdonald et al., 2014). Therefore, the response to selection in RFI presented here could, in fact, be conservative. Furthermore, although lactation is the life stage with a larger effect on lifetime income, rearing is also an important life stage because minimizing its cost has an effect on lifetime profitability. In fact, the economic value for RFI (calculated as an annualized value) in this study included both aspects: heifer and cow RFI weighted for the proportion of life at these stages. An alternative and interesting scenario could be to split RFI into 2 life stages and explore the consequences of selecting for these traits separately.

It is worth pointing out that the use of DMI in the selection index instead of RFI should lead to similar results, assuming that proper economic values and genomic correlations have been estimated. If DMI is included in the index, the correlations with other traits are accounted for within the index calculations, whereas if RFI is included, some of these correlations (e.g., with production and growth) are preadjusted for.

Genetic correlation estimates between RFI, fertility, and health traits should be monitored in the future as data cumulates. Selection for RFI is associated with a lower BCS, which is known to be unfavorably correlated with fertility and health traits (Berry et al., 2014). Although the weight applied to RFI was low in the selection index of this study, an increase in the reliability of genomic heifer RFI may occur during the next few years and its implications on correlated traits must be evaluated routinely to continue to ensure that health and reproduction traits are not compromised.

\section{CONCLUSIONS}

The inclusion of RFI for growing heifers in the calculation of the APR index could increase the annual genetic gain of farm profitability by $3 \%$, to $\mathrm{A} \$ 23.28$ / 

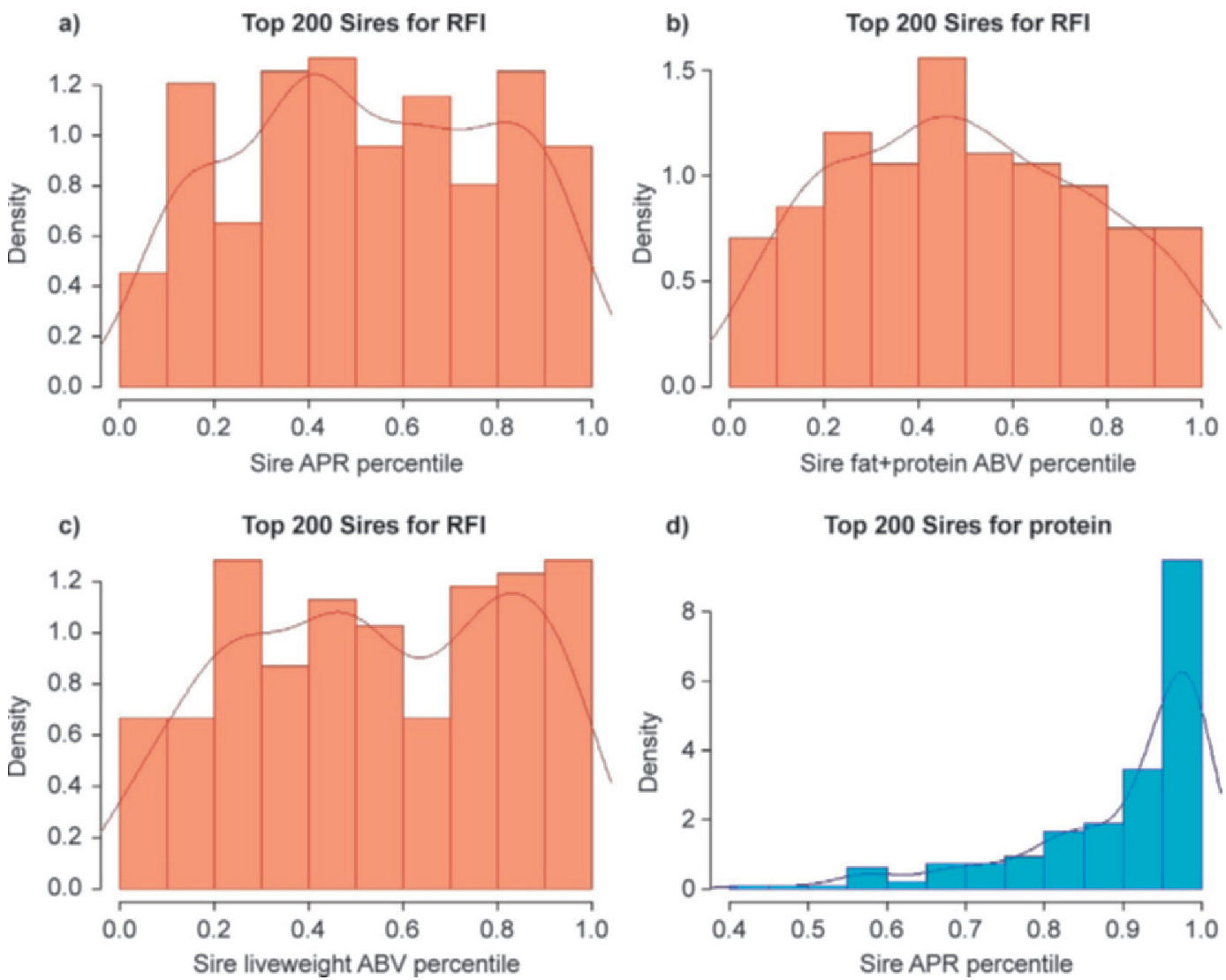

Figure 3. Percentile of Australian Profit Ranking (APR; a), fat + protein predicted breeding value (b), and liveweight breeding value (c) for sires in the 97th percentile for residual feed intake (RFI; top 200 animals). The APR percentile for sires in the 97th percentile for protein breeding values is in (d). ABV $=$ Australian breeding value. Color version available in the online PDF.

cow per year, which equates to an industry-wide gain of $\mathrm{A} \$ 0.55$ million in the Australian Holstein population, given the number of cows and assuming $100 \%$ uptake of the trait. The effect of including RFI in the selection index is to reduce RFI while achieving similar productivity. Caution should be placed on the antagonism between RFI and ClvI, with enough weight on fertility in the index to preclude unfavorable selection responses. This study shows that even with the antagonistic correlation between RFI and fertility that was estimated from our data $(-0.13)$, the extended APR index would select more feed-efficient progeny without impairing fertility in Holstein cows. Including RFI as part of a multitrait selection index is the obvious way to achieve optimal responses to selection in all traits of economic importance while avoiding unfavorable responses to selection that may arise as a consequence of selecting on RFI independently. Inclusion of RFI in the selection index for dairy cattle will be a small but significant contribution toward producing more highquality protein from fewer resources to meet project increases in global demand.

\section{ACKNOWLEDGMENTS}

Julius van der Werf (University of New England, Armidale, Australia) is gratefully acknowledged for his work in developing the software used for calculating responses to selection on the Australian Profit Ranking index. The authors thank the Gardiner Foundation (Melbourne, Australia) and Dairy Futures Cooperative Research Centre (Melbourne, Australia) for funding this research.

\section{REFERENCES}

Arthur, P. F., J. A. Archer, D. J. Johnston, R. M. Herd, E. C. Richardson, and P. F. Parnell. 2001. Genetic and phenotypic variance and covariance components for feed intake, feed efficiency, and other postweaning traits in Angus cattle. J. Anim. Sci. 79:2805-2811.

Bell, M. J., R. J. Eckard, M. Haile-Mariam, and J. E. Pryce. 2013. The effect of changing cow production and fitness traits on net income and greenhouse gas emissions from Australian dairy systems. 96:7918-7931. J. Dairy Sci.

Berry, D. P., M. P. Coffey, J. E. Pryce, Y. de Haas, P. Løvendahl, N. Krattenmacher, J. J. Crowley, Z. Wang, D. Spurlock, K. Weigel, K. Macdonald, and R. F. Veerkamp. 2014. International genetic evaluations for feed intake in dairy cattle through the collation of data from multiple sources. J. Dairy Sci. 97:3894-3905. http:// dx.doi.org/10.3168/jds.2013-7548. 
Browning, B. L., and S. R. Browning. 2009. A unified approach to genotype imputation and haplotype-phase inference for large data sets of trios and unrelated individuals. Am. J. Hum. Genet. 84:210-223.

de Haas, Y., M. P. L. Calus, R. F. Veerkamp, E. Wall, M. P. Coffey, H. D. Daetwyler, B. J. Hayes, and J. E. Pryce. 2012. Improved accuracy of genomic prediction for dry matter intake of dairy cattle from combined European and Australian data sets. J. Dairy Sci. 95:6103-6112.

Erbe, M., B. J. Hayes, L. K. Matukumalli, S. Goswami, P. J. Bowman, and C. M. Reich. 2012. Improving accuracy of genomic predictions within and between dairy cattle breeds with imputed high-density single nucleotide polymorphism panels. J. Dairy Sci. 95:41144129 .

Garrick, D. J., J. F. Taylor, and R. L. Fernando. 2009. Deregressing estimated breeding values and weighting information for regression analysis. Genet. Sel. Evol. 41:55.

Geyer, C. J. 1992. Practical Markov chain Monte Carlo. Stat. Sci. 7:473-511.

Haile-Mariam, M., P. J. Bowman, and J. Pryce. 2013a. Genetic analyses of fertility in Holstein herds with low and high mean calving intervals and in Jersey herds. J. Dairy Sci. 96:655-667.

Haile-Mariam, M., O. Gonzalez-Recio, and J. E. Pryce. 2014. Prediction of liveweight of cows from type traits and its relationship with production and fitness traits. J. Dairy Sci. 97:3173-3189.

Haile-Mariam, M., G. J. Nieuwhof, K. T. Beard, K. V. Konstantinov, and B. J. Hayes. 2013b. Comparison of heritabilities of dairy traits in Australia Holstein-Friesian cattle from genomic and pedigree data and implications for genomic evaluations. J. Anim. Breed. Genet. 130:20-31.

Hayes, B. J., H. A. Lewin, and M. E. Goddard. 2013. The future of livestock breeding: Genomic selection for efficiency, reduced emissions intensity, and adaptation. Trends Genet. 29:206-214.

Hazel, L. N. 1943. The genetic basis for constructing selection indices. Genetics 28:476-490.

Ho, C., R. Nessler, P. Doyle, and B. Malcolm. 2005. Future dairy farming systems in irrigating regions. AFBM J. 2:59-68.

Macdonald, K. A., J. E. Pryce, R. J. Spelman, S. R. Davis, W. J. Wales, G. C. Waghorn, Y. J. Williams, L. C. Marett, and B. J. Hayes. 2014. Holstein-Friesian calves selected for divergence in residual feed intake during growth also exhibit divergence in residual feed intake in their first lactation. J. Dairy Sci. 97:1427-1435.

Meuwissen, T. H. E., B. J. Hayes, and M. E. Goddard. 2001. Prediction of total genetic value using genome-wide dense marker maps. Genetics 157:1819-1829.

Nieuwhof, G. J., J. A. Van Arendonk, H. Vos, and S. Korver. 1992. Genetic relationships between feed intake, efficiency and production traits in growing bulls, growing heifers and lactating heifers. Livest. Prod. Sci. 32:189-202.
Pryce, J. E., J. Arias, P. J. Bowman, S. R. Davis, K. A. Macdonald, G. C. Waghorn, W. J. Wales, Y. J. Williams, R. J. Spelman, and B. J. Hayes. 2012. Accuracy of genomic predictions of residual feed intake and 250-day body weight in growing heifers using 625,000 single nucleotide polymorphisms markers. J. Dairy Sci. 95:21082119.

Pryce, J. E., M. E. Goddard, H. W. Raadsma, and B. J. Hayes. 2010. Deterministic models of breeding scheme designs that incorporate genomic selection. J. Dairy Sci. 93:5455-5466.

Pryce, J. E., O. Gonzalez-Recio, J. B. Thornhill, L. C. Marett, W. J. Wales, M. P. Coffey, Y. de Haas, R. F. Veerkamp, and B. J. Hayes. 2014a. Short communication: Validation of genomic breeding value predictions for feed intake and feed efficiency traits. J. Dairy Sci. 97:537-542.

Pryce, J. E., J. H. J. van der Werf, M. Haile-Mariam, B. Malcolm, and M. E. Goddard. 2009. Updated index weights for the Australian Profit Ranking in dairy cattle. Proc. Adv. Anim. Breed. Genet. $18: 143-146$

Pryce, J. E., W. J. Wales, Y. de Haas, R. F. Veerkamp, and B. J. Hayes. 2014b. Invited review: Genomic selection for feed efficiency in dairy cattle. Animal 8:1-10.

Shaffer, K. S., P. Turk, W. R. Wagner, and E. E. D. Felton. 2011. Residual feed intake, body composition, and fertility in yearling beef heifers. J. Anim. Sci. 89:1028-1034.

Simm, G., R. F. Veerkamp, and P. Persaud. 1994. The economic performance of dairy cows of different predicted genetic merit for milk solids production. Anim. Prod. 58:313-320.

United Nations, Department of Economics and Social Affairs, Population Division. 2010. World population prospects: The 2010 Revision, Volume II: Demographic profiles. http://esa.un.org/wpp/ Documentation/publications.htm.

Vallimont, J. E., C. D. Dechow, J. M. Daubert, M. W. Dekleva, J. W. Blum, W. Liu, G. A. Varga, A. J. Heinrichs, and C. R. Baumrucker. 2013. Short communication: Feed utilization and its associations with fertility and productive life in 11 commercial Pennsylvania tie-stall herds. J. Dairy Sci. 96:1251-1254.

Veerkamp, R. F., J. K. Oldenbroek, H. J. Van der Gaast, and J. H. J. Van der Werf. 2000. Genetic correlation between days until start of luteal activity and milk yield, energy balance, and live weights. J. Dairy Sci. 83:577-583.

Williams, Y. J., J. E. Pryce, C. Grainger, W. J. Wales, N. Linden, M. Porker, and B. J. Hayes. 2011. Variation in residual feed intake in Holstein Friesian dairy heifers in southern Australia. J. Dairy Sci. 94:4715-4725.

Yang, J., B. Benyamin, B. P. McEvoy, S. Gordon, A. K. Henders, D. R. Nyholt, P. A. Madden, A. C. Heath, N. G. Martin, G. W. Montgomery, M. E. Goddard, and P. M. Visscher. 2010. Common SNPs explain a large proportion of the heritability for human height. Nat. Genet. 42:565-569. 